\title{
Neurosarcoidosis Characterized by Necrotizing Granulomas: The Challenges and Pitfalls of Diagnosing Neurosarcoidosis
}

\author{
Jeffrey Harte ${ }^{1, *}$, Chloe Doran ${ }^{1}$, Alan Beausang ${ }^{2}$, Cora McNally ${ }^{1}$ \\ ${ }^{1}$ Department of Infectious Disease, Beaumont Hospital, Dublin, Ireland \\ ${ }^{2}$ Department of Pathology, Beaumont Hospital, Dublin, Ireland
}

*Corresponding author: Jeffrey Harte, Department of Infectious Disease, Beaumont Hospital, Dublin, Ireland; E-mail: ohairts@ tcd.ie

Received Date: January 22, 2019 Accepted Date: February 28, 2019 Published Date: March 05, 2019

Citation: Jeffrey Harte (2019) Neurosarcoidosis Characterized by Necrotizing Granulomas: The Challenges and Pitfalls of Diagnosing Neurosarcoidosis. Case Reports: Open Access 3: 1-3.

\section{Summary}

A 58-year-old woman presented to her local hospital with a 2-week duration of headaches, fatigue and new onset ataxia due to obstructive hydrocephalus. Biopsy of the obstructing lesion in the third ventricle demonstrated necrotizing granuloma. She was initiated on anti-tuberculosis (TB) medications and steroids for TB meningitis (TBM). She noted a relapse of symptoms following discontinuation of steroids. Upon completion of therapy and recurrence of symptoms, positron emission tomography (PET) scan showed widespread lymphadenopathy and she is being treated now as a case of neuro-sarcoidosis.

\section{Background}

Central nervous system TB is difficult to diagnose on cerebrospinal fluid sampling and is often treated empirically. Sarcoidosis is a common mimic of TB.

\section{Case presentation}

A 58-year-old woman presented to her local hospital with a 2 -week duration of headaches, fatigue, and new onset ataxia. She also noted ongoing night sweats and weight loss prior to this presentation. She had no history of respiratory symptoms, no sick contacts, and no significant past medical history. On examination, she exhibited no signs of meningism or focal neurology

\section{Investigations}

Her hematological results were all within the normal range. Her serum calcium and angiotensin-converting enzyme level were normal. Her chest x-ray showed no evidence of hilar lymphadenopathy or upper lobe calcifications. A noncontrast CT head was performed which showed hydrocephalus caused by an obstructing lesion in the third ventricle. She was transferred to our hospital for placement of an external ventricular drain and a biopsy. The resultant biopsy showed necrotizing granulomatous inflammation (Figure 1) and stated tuberculosis was the likely cause, however with a caveat that rarely necrosis could be present in sarcoidosis-associated granulomas. Following the procedure, she still reported profound fatigue, chronic headaches, and night sweats. Analysis of her CSF demonstrated lymphocytic meningitis, low CSF: plasma glucose ratio with elevated protein. Tuberculosis was not identified on skin testing, interferon testing, cultures or PCR. She had an MRI brain which demonstrated leptomeningeal enhancement across the pons (Figure 2). This was reported as in keeping with basal meningitis and tuberculosis. 


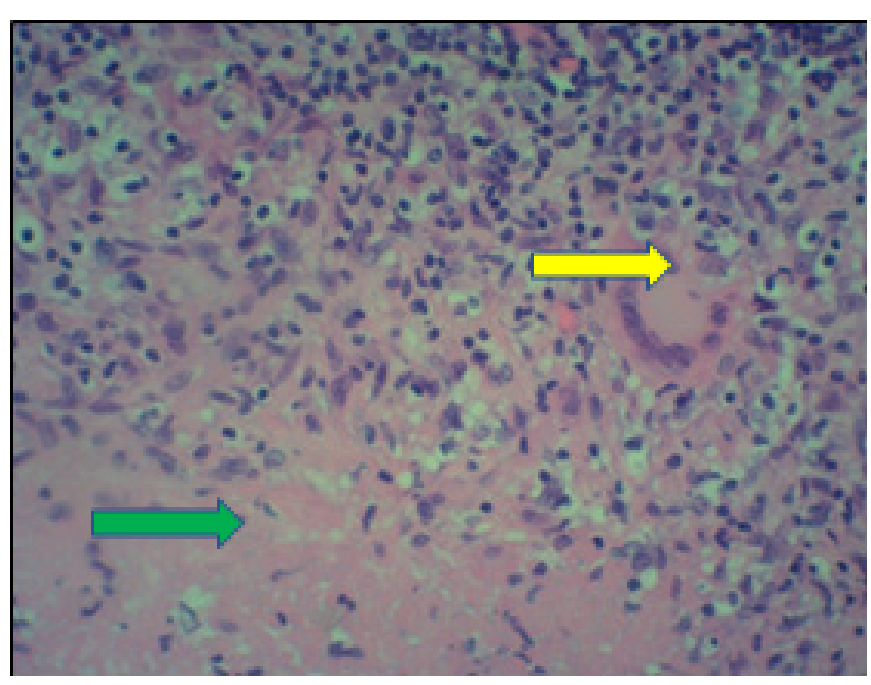

Figure 1. Yellow arrow $=$ multinucleated giant cell. Green arrow $=$ necrosis

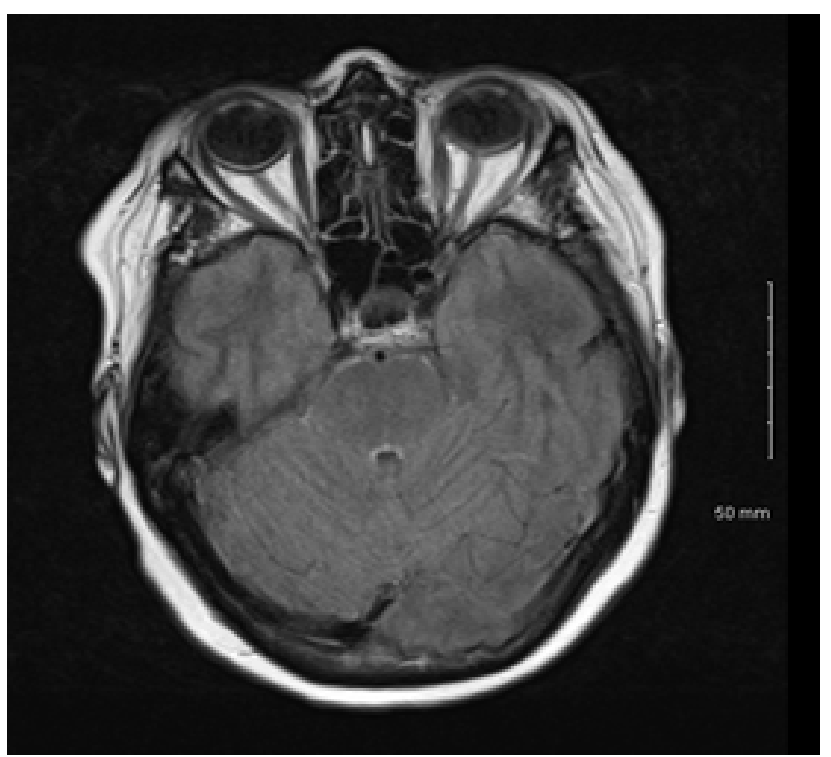

Figure 2. Faint FLAIR hyperintensity and thin leptomeningeal enhancement along the surface of the pons.

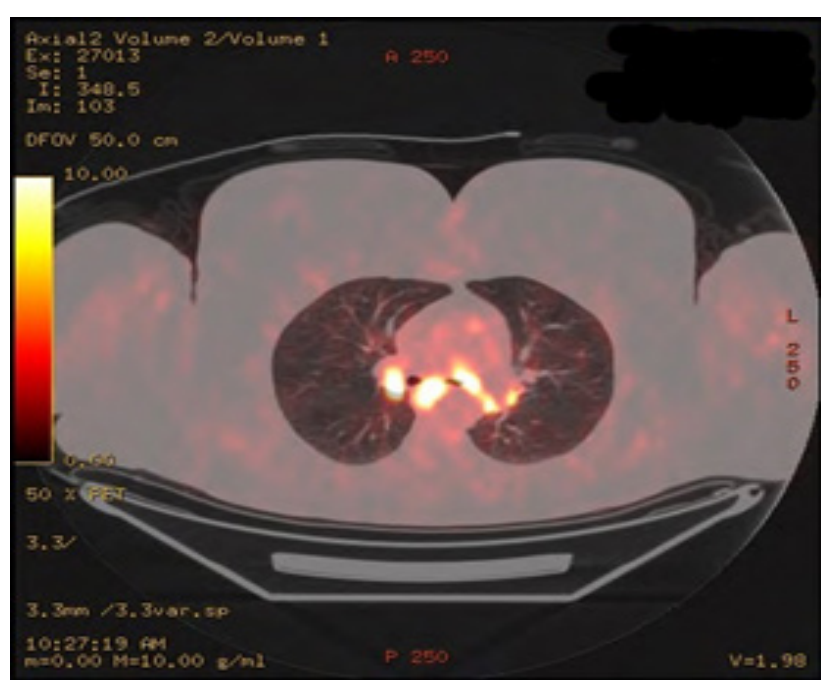

Figure 3. FDG avid nodes in essentially all mediastinal stations.

\section{Treatment}

She was initiated on anti-tuberculosis medications and high dose steroids. The patient noted a marked improvement in symptomatology in her early weeks of treatment. She was seen in outpatient clinic two months into her treatment who noted dis-improvement upon discontinuation of oral steroids following dose tapering. She was treated for a sixteen-month course for TBM and was monitored frequently. Upon returning to the clinic at completion the patient reportedly felt no different to her presentation 18 months ago. A further LP showed an ongoing lymphocytic and raised protein CSF. An MRI brain performed at this time showed no improvement from prior imaging with persistent leptomeningeal enhancement. The patient also had a PET scan at this time which showed FDG uptake in multiple nodes in her mediastinum (Figure 3) The patient had a subcarinal lymph node biopsy which showed non-caseating granulomas. She is now being treated for systemic sarcoidosis.

\section{Discussion}

TBM most often presents with insidious onset headaches and often with evidence of tuberculomas [1]. Neurosarcoid most commonly presents with cranial neuropathies and other symptoms of systemic disease [2]. TBM mortality exceeds 50\%(1), however, diagnosis of central nervous system tuberculosis has very poor sensitivity. CSF smear microscopy for AFB has $<15 \%$ sensitivity [3]. Mycobacteria culture is better with a sensitivity of 50-60\% [4], however, there is a significant delay in its growth taking up to 10 days on liquid media. Often clinical decision making cannot wait this long. PCR sensitivity is approximately 60\% in TBM, however, this can be maximized to $70-80 \%$ with large volumes of CSF, which is often impractical. Due to these weaknesses in diagnostics, necrotizing granulomas, lymphocytic CSF and leptomeningeal enhancement on MRI, it is reasonable to have initiated this patient on anti-TB medications in view of her possible diagnosis. Delayed diagnosis and treatment are associated with poor prognosis and death in TBM [5]. British Infection Society guidelines recommend initiating empiric anti-TB therapy promptly in suspected cases [5]. Steroids have been shown to increase survival in TBM.

Necrotizing granulomas are classically associated with $\mathrm{TB}$, however, can occasionally occur in the setting of sarcoidosis. A neurosarcoidosis diagnosis would be consistent with the patient's CSF findings and MRI findings, however, her biopsy results and clinical presentation would be a more 
atypical presentation [2]. Her response to steroids, persistent symptoms, serum and imaging findings with anti-TB therapy and the subcarinal biopsy showing non-caseating granulomas are more in keeping with neurosarcoidosis.

\section{Learning points}

Testing sensitivity for TBM is poor.

$>$ Empiric treatment for TBM is advised in suspected cases.

$>$ Neuro-sarcoidosis can mimic TBM.

$>$ Clinical response to treatment is an essential component of optimal neurosarcoidosis treatment.

\section{Contributors}

Clinical work-up and all the work surrounding the write-up of this case have been under the stewardship of CM. CM is the acting guarantor for this work. $\mathrm{JH}$ is an Infectious Disease intern at Beaumont Hospital Dublin. He reviewed the patient on the ward, with $\mathrm{CD}$, has followed the patient's investigation results, performed a literature search on this topic and wrote the case report under the guidance of CM. The histopathology images have been supplied by the histopathology department in Beaumont Hospital.

\section{Funding}

This research received no specific grant from any funding agency in the public, commercial or not-for-profit sectors.

Provenance and peer review Not commissioned; externally peer reviewed.

Author note This case was presented to the Department of Infectious Disease in Beaumont Hospital. This patient is under the care of CM, Consultant in Infectious Disease.

\section{References}

1. Bahr N, Nuwagira E, Evans E, Cresswell F, Bystrom P, et al. (2018) Diagnostic accuracy of Xpert MTB/RIF Ultra for tuberculous meningitis in HIV-infected adults: a prospective cohort study. The Lancet Infectious Diseases. 18:68-75.

2. Ibitoye RT, Wilkins A (2017) Scolding NJ.Neurosarcoidosis: a clinical approach to diagnosis and management. Journal of Neurology 264:1023-1028.

3. Dheda K, Barry C (2016) Maartens G. Tuberculosis. The Lancet 387:1211-1226.

4. Torok M (2015) Tuberculous meningitis: advances in diagnosis and treatment. British Medical Bulletin 113:117131.

5. Wilkinson R, Rohlwink U, Misra U, van Crevel R, et al. (2017) Tuberculous meningitis. Nature Reviews Neurology 13:581-598.

6. Thwaites G, Fisher M, Hemingway C, Scott G, Solomon T, et al. (2009) British Infection Society guidelines for the diagnosis and treatment of tuberculosis of the central nervous system in adults and children. Journal of Infection 59, 167187.

Submit your manuscript to a JScholar journal and benefit from:

ब Convenient online submission

- Rigorous peer review

- Immediate publication on acceptance

ब Open access: articles freely available online

q. High visibility within the field

ब Better discount for your subsequent articles

Submit your manuscript at

http://www.jscholaronline.org/submit-manuscript.php 